\title{
Severe, But Not Moderate Asthmatics Share Blood Transcriptomic Changes With Post-Traumatic Stress Disorder And Depression.
}

Sandor Haas-Neill ( $\square$ haasneis@mcmaster.ca )

Brain Body Institute, St. Joseph's Hospital, Hamilton

Anna Dvorkin-Gheva

McMaster University, Hamilton, Ontario, Canada

Paul Forsythe

University of Alberta

\section{Research Article}

Keywords: asthma, disorders, stress, Gene

Posted Date: December 29th, 2021

DOI: https://doi.org/10.21203/rs.3.rs-1193123/v1

License: (c) (1) This work is licensed under a Creative Commons Attribution 4.0 International License. Read Full License 


\section{Abstract}

Asthma, an inflammatory disorder of the airways, is one of the most common chronic illnesses worldwide and is associated with significant morbidity. There is growing recognition of an association between asthma and mood disorders including post-traumatic stress disorder (PTSD) and major depressive disorder (MDD). Although there are several hypotheses regarding the relationship between asthma and mental health, there is little understanding of underlying mechanisms and causality. In the current study we utilized publicly available datasets of human blood mRNA collected from patients with severe and moderate asthma, MDD, and PTSD. We performed differential expression (DE) analysis and Gene Set Enrichment Analysis (GSEA) on diseased subjects against the healthy subjects from their respective datasets, compared the results between diseases, and validated DE genes and gene sets with 4 more independent datasets. Our analysis revealed that commonalities in blood transcriptomic changes were only found between the severe form of asthma and mood disorders. Gene expression commonly regulated in PTSD and severe asthma, included ORMDL3 a gene known to be associated with asthma risk and STX8, which is involved in TrkA signalling. We also identified several pathways commonly regulated to both MDD and severe asthma. This study reveals gene and pathway regulation that potentially drives the comorbidity between severe asthma, PTSD, and MDD and may serve as foci for future research aimed at gaining a better understanding of both the relationship between asthma and PTSD, and the pathophysiology of the individual disorders.

\section{Introduction:}

Asthma is a chronic inflammatory disease of the airways associated with recurrent episodes of wheezing, shortness of breath, chest tightness, and coughing. Generally, asthma is characterized by reversible constriction of the airways in response to allergen, but it can also be triggered by viral infection, physical activity, stress, or a negative $\operatorname{mood}^{1}$. Asthma affects 300 million people worldwide and the World Health Organization has estimated that it is responsible for the loss of 15 million disability-adjusted life years (DALYs) annually ${ }^{2,3}$. Asthma is also the most common chronic disease in children ${ }^{4}$.

Epidemiological studies have shown significant association between asthma and mental health disorders, including anxiety, depression, panic attacks, and posttraumatic stress disorder (PTSD) $)^{5,6,7,8,9,10}$.

MDD, more commonly referred to as 'depression' is a mental health disorder characterized by a low self-esteem, mood, and enjoyment of activities ${ }^{11}$.

Studies have demonstrated consistent comorbidity between asthma and depression ${ }^{7}$ and Youth with asthma are close to twice as likely to have anxiety and depressive disorders as those without asthma ${ }^{8}$. The co-occurrence of an anxiety or depressive disorder is associated with poor symptom control, impaired quality of life and increased health care utilization. While many studies have focused on psychosocial factors linking asthma and depression there is evidence that there may be shared pathophysiological factors between the diseases. For example, in a large-scale study in adults twins the association between depression and asthma remained significant after controlling for genetic and environmental factors ${ }^{12}$. However, the potential mechanisms and causality relating depression and asthma remain unclear ${ }^{13,14,15}$

PTSD is a mental health disorder that usually follows exposure to a traumatic event. The characteristic symptoms of PTSD include intrusive memories and nightmares, negative mood impaired cognition, avoidance behaviours, and changes to arousal behaviours such as increased irritability ${ }^{16}$.

Clinical evidence supports a strong link between inflammatory conditions and PTSD with a particularly strong association between asthma and the prevalence and severity of PTSD ${ }^{17,18}$. A twin study of Vietnam war veterans found that those with the top quartile of PTSD scores were 2-fold more likely to have asthma than those in the lower quartile ${ }^{6}$. This association was shown not to be predicted by familial or genetic factors, smoking, depression, or demographic factors ${ }^{6}$. Wisnivesky et al., $(2021)^{5}$ found that $19 \%$ of world trade center rescue and recovery workers with asthma also had PTSD, 10 times the prevalence in the general population. PTSD is also one of the greatest risk factors for decreased quality-of-life related to asthma $^{17,18}$ and these poorer asthma outcomes do not appear to be due to differences in key asthma self-management behaviors ${ }^{18}$. Conversely, individuals with asthma prior to PTSD have been demonstrated to develop more aggravated asthma symptoms after the development of PTSD, while non-asthmatic subjects who develop PTSD have increased risk of adult onset asthma, suggesting a bidirectional relationship between these disorders ${ }^{17}$.

An attempt by Jiang et al., $(2014)^{7}$ to identify a mechanism behind the comorbidity of asthma and MDD suggested immune factors may underlie both disorders. The investigation of 38 depression studies found that monocyte-derived, and other inflammatory cytokines (IL-1, IL-4, IL-6, and TNF) were significantly overexpressed in individuals with depression, while T cell derived cytokines (IL-10, and INF- $\mathbf{Y}$ ) were uncorrelated with depression.

Genome-wide association studies (GWAS) have identified some shared genetic traits between those with asthma and MDD ${ }^{19,20}$. In a cross-trait metaanalysis, Zhu et al. (2019) ${ }^{19}$ identified 10 genomic loci shared between asthma and MDD and mendelian randomization identified a significant causal effect of MDD on asthma. The cross-trait meta-analysis performed by Cao et al., $(2021)^{20}$ identified 18 loci jointly associated between MDD and atopic diseases (asthma, eczema, and hay fever). Through Mendelian randomization analysis the investigators found that MDD confers a 
stronger causal effect on those atopic diseases than they confer on MDD. Similarly, in a meta-analysis by Nievergelt et al., $(2019)^{21}$, a pairwise genetic correlation demonstrated a high association between PTSD and asthma.

With a deeper understanding of the established comorbidity between mental health disorders and asthma, may come tangible knowledge on how to combat the root cause of these diseases and an expectation for how treatment of one disorder might affect another. Therefore, the goal of this study was to expand on genome-wide association studies by using publicly available data to characterize transcriptomic similarities between these disorders through analysis of genes and gene sets commonly differentially expressed between those suffering from the diseases and healthy subjects.

\section{Results:}

\section{Exploration of Commonly Differentially Expressed Genes:}

The 3 exploration datasets underwent principal component analysis to check that no known variables could account for major differences that may arise during DE and GSEA analysis (Figure 1). There was no apparent grouping along PC1 or PC2 for any of the datasets, including for diseased vs healthy subjects (Figure 1). For the PTSD exploration cohort, $40.7 \%$ of the variance was accounted for by PC1, and $10.6 \%$ by PC2; for MDD, $19.0 \%$ of the variance was accounted for by PC1, and $6.9 \%$ by PC2; and for asthma, $22.7 \%$ of the variance was explained by PC1, and $10.0 \%$ by $\mathrm{PC} 2$.

Differential expression analysis of each disease to control subjects from their respective datasets reveals significant differences in both genes being up- and downregulated in all diseases except for MDD (Figure 2). The analysis identified 8,321, 1,736, and 373 genes significantly upregulated (adjusted p-value < 0.05; FC $\geq 1.5$ ) in PTSD, severe asthma, and moderate asthma respectively, as compared to the corresponding controls. 7,062, 2,735 , and 901 genes were found to be significantly downregulated (adjusted p-value $<0.05 ; \mathrm{FC} \leq-1.5$ ) in the same comparisons respectively. No genes appeared to be significantly DE in the MDD exploration dataset.

Lists of significantly regulated (adjusted P-value $<0.05,|F C| \geq 1.5$ ) genes were compared between the exploration datasets for each disease. Genes found commonly to be regulated in the same direction in patients relatively to the healthy controls for multiple diseases were tallied in (Table 1 ). A detailed list of all the genes in Table 1 can be found in (Supplementary Table 1).

Table 1

The number of genes differentially expressed from healthy subjects in the same direction between different diseases.

\begin{tabular}{|lll|}
\hline Expression commonly DE from Healthy & Upregulated Genes & Downregulated Genes \\
\hline PTSD, Moderate Asthma, Severe Asthma & 47 & 76 \\
\hline PTSD, Severe Asthma & 355 & 255 \\
\hline PTSD, Moderate Asthma & 24 & 27 \\
\hline Moderate Asthma, Severe Asthma & 197 & 506 \\
\hline
\end{tabular}

\section{Exploration of Commonly Regulated Gene Sets:}

To detect the biological effect of more nuanced changes in all disease groups, Gene Set Enrichment Analysis (GSEA) was performed. GSEA compared expression of selected lists of genes (here termed "gene sets") between diseased and healthy subjects in each dataset (Figure 3 ). No Hallmark gene sets were enriched in the same direction between all 4 datasets. $3 \mathrm{C} 2$ gene sets were found to be upregulated in all 4 groups: REN_ALVEOLAR_RHABDOMYOSARCOMA_DN, JISON_SICKLE_CELL_DISEASE_UP, and REACTOME_NEUTROPHIL_DEGRANULATION. No C2 gene sets were commonly downregulated in all 4 groups. $55 \mathrm{C} 7$ gene sets were commonly upregulated in all 4 groups, but nothing was commonly downregulated in all 4 of those groups.

\section{Validation of Differentially Expressed Genes in independent transcriptomic datasets:}

To challenge these findings, the 'validation' datasets for each of: MDD, PTSD, severe asthma, and moderate asthma underwent DE analysis with limma. No genes were found to be significantly regulated (adjusted P-value $<0.05$ ) in the same directions for all 4 sets as no individual genes were significantly differentially expressed in either of the MDD exploration/validation datasets. 2 genes were validated as upregulated in PTSD and severe asthma: STX8 and ARHGAP24. Commonly downregulated to PTSD and severe asthma were ORMDL3, PTP4A3, SHISA4, and TPPP3. No differentially expressed genes were validated between PTSD and moderate asthma in either direction, however. 582 genes were validated as significantly downregulated between moderate and severe asthma while no upregulated genes could be validated.

Validation of Regulated Pathways in independent transcriptomic datasets: 
The same datasets used to validate differentially expressed genes were used to validate gene sets and pathways identified as being commonly regulated in either direction in the exploration datasets. Interestingly, despite no genes being significantly differentially expressed in MDD patients vs healthy controls in either dataset, there were pathways identified as being significantly altered in severe asthma patients as compared to their corresponding controls (Table 2).

Table 2

Directionally validated pathway comparisons in the C7 collection following GSEA excluding comparison between severe and moderate asthma.

\begin{tabular}{|c|c|c|c|}
\hline Direction & Comparison & Enriched Gene Set & $\begin{array}{l}\text { Adjusted } \\
\text { Adjusted } \\
\text { P-Values }\end{array}$ \\
\hline Up & $\begin{array}{l}\text { MDD vs } \\
\text { Severe } \\
\text { Asthma }\end{array}$ & GSE4748_CYANOBACTERIUM_LPSLIKE_VS_LPS_AND_CYANOBACTERIUM_LPSLIKE_STIM_DC_3H_DN & $\begin{array}{l}\text { MDD1 - } \\
\text { 1.58e-11, } \\
\text { MDD2 - } \\
\text { 1.30e-2, } \\
\text { S.Asthma1 } \\
\text { - 5.49e-14, } \\
\text { S.Asthma2 } \\
-3.42 \mathrm{e}-12\end{array}$ \\
\hline & & GSE34205_HEALTHY_VS_RSV_INF_INFANT_PBMC_DN & $\begin{array}{l}\text { MDD1 - } \\
\text { 7.69e-12, } \\
\text { MDD2 - } \\
1.16 \mathrm{e}-2 \text {, } \\
\text { S.Asthma1 } \\
\text { - } 1.79 \mathrm{e}-10 \text {, } \\
\text { S.Asthma2 } \\
-6.29 \mathrm{e}-10\end{array}$ \\
\hline Down & $\begin{array}{l}\text { MDD vs } \\
\text { Severe } \\
\text { Asthma }\end{array}$ & GSE22886_NAIVE_BCELL_VS_NEUTROPHIL_UP & $\begin{array}{l}\text { MDD1 - } \\
1.30 \mathrm{e}-10, \\
\\
\text { MDD2 - } \\
2.61 \mathrm{e}-3 \text {, } \\
\text { S.Asthma1 } \\
-4.41 \mathrm{e}-7 \text {, } \\
\text { S.Asthma2 } \\
-2.27 \mathrm{e}-9\end{array}$ \\
\hline & & GSE34205_HEALTHY_VS_FLU_INF_INFANT_PBMC_UP & $\begin{array}{l}\text { MDD1 - } \\
4.69 \mathrm{e}-3, \\
\text { MDD2 - } \\
2.26 \mathrm{e}-2 \text {, } \\
\text { S.Asthma1 } \\
\text { - 4.47e-3, } \\
\text { S.Asthma2 } \\
\text { - 9.99e-6 }\end{array}$ \\
\hline & & GSE22886_NEUTROPHIL_VS_MONOCYTE_DN & $\begin{array}{l}\text { MDD1 - } \\
3.37 \mathrm{e}-2, \\
\text { MDD2 - } \\
\text { 7.46e-8, } \\
\text { S.Asthma1 } \\
\text { - 3.74e-2, } \\
\text { S.Asthma2 } \\
-3.40 \mathrm{e}-3\end{array}$ \\
\hline
\end{tabular}

As may be expected, many pathways were found to be commonly modified between moderate and severe asthma when comparing against the C2 and C7 gene sets and they can be found listed in supplementary information (Supplementary Table 2). Barcode plots showing a more detailed crosssection of gene expression from the sets in Table 3 can be found in supplementary information (Supplementary Figure 1-5). 
Table 3

List of datasets used in this paper with a description of data type, preprocessing, number of genes and gene variants remaining in the dataset following preprocessing, and associated publications.

\begin{tabular}{|c|c|c|c|c|c|c|c|}
\hline GSE \# & $\begin{array}{l}\text { Platform } \\
\text { (GPL) }\end{array}$ & $\begin{array}{l}\text { Source and } \\
\text { Species }\end{array}$ & $\begin{array}{l}\text { Normalization } \\
\text { Method }\end{array}$ & Purpose & $\begin{array}{l}\text { Associated } \\
\text { Publication }\end{array}$ & \# Samples & $\begin{array}{l}\text { \# Genes / } \\
\text { Variants }\end{array}$ \\
\hline GSE81761 & $\begin{array}{l}\text { GPL570 } \\
\text { (Array) }\end{array}$ & $\begin{array}{l}\text { Human whole } \\
\text { blood mRNA }\end{array}$ & RMA & PTSD Exploration & $\begin{array}{l}\text { Rusch et al., } \\
2019\end{array}$ & $\begin{array}{l}27 \text { - PTSD, } \\
39 \text { - No PTSD }\end{array}$ & 44,134 \\
\hline GSE97356 & $\begin{array}{l}\text { GPL11154 } \\
\text { (RNAseq) }\end{array}$ & $\begin{array}{l}\text { Human whole } \\
\text { blood mRNA }\end{array}$ & TMM & PTSD Validation & $\begin{array}{l}\text { Kuan et al., } \\
2017\end{array}$ & $\begin{array}{l}82 \text { - PTSD, } \\
201 \text { - No PTSD }\end{array}$ & 15,112 \\
\hline GSE98793 & $\begin{array}{l}\text { GPL570 } \\
\text { (Array) }\end{array}$ & $\begin{array}{l}\text { Human whole } \\
\text { blood RNA }\end{array}$ & RMA & MDD Exploration & $\begin{array}{l}\text { Leday et al., } \\
2018\end{array}$ & $\begin{array}{l}64 \text { - MDD, } \\
32 \text { - No MDD }\end{array}$ & 44,134 \\
\hline GSE19738 & $\begin{array}{l}\text { GPL6848 } \\
\text { (Array) }\end{array}$ & $\begin{array}{l}\text { Human whole } \\
\text { blood RNA }\end{array}$ & Quantile & MDD Validation & $\begin{array}{l}\text { Spijker et al., } \\
2010\end{array}$ & $\begin{array}{l}33 \text { - MDD, } \\
34 \text { - No MDD }\end{array}$ & 12,816 \\
\hline GSE69683 & $\begin{array}{l}\text { GPL13158 } \\
\text { (Array) }\end{array}$ & $\begin{array}{l}\text { Human whole } \\
\text { blood RNA }\end{array}$ & RMA & $\begin{array}{l}\text { Asthma Exploration } \\
\text { and Validation }\end{array}$ & $\begin{array}{l}\text { Bigler et al., } \\
2017\end{array}$ & $\begin{array}{l}\text { After Split: } \\
\text { Exploration: } \\
58 \text { - Healthy, } \\
58 \text { - Moderate, } \\
216 \text { - Severe. } \\
\text { Validation: } \\
28 \text { - Healthy, } \\
20 \text { - Moderate, } \\
128 \text { - Severe }\end{array}$ & 41,791 \\
\hline
\end{tabular}

\section{Discussion:}

While it is widely accepted that psychosocial factors affect asthma pathobiology in children and adults, there is little understanding of potential common biological pathways underlying comorbidity between asthma and mental health disorders. Previous reports based on GWAS studies were focusing on determination of shared genetic traits between Asthma, MDD and PTSD or on circulating levels of specific inflammatory cytokines to explore potential shared pathophysiology of these disorders. In an attempt to provide further insight into the comorbidity of these conditions and to identify target pathways for further investigation, we utilized publicly available data to assess similarities between asthma, MDD and PTSD at the transcriptomic level.

As expected, asthma, MDD and PTSD were associated with many differentially expressed genes and gene sets, and, in comparing exploration cohorts, a large number of gene sets were significantly regulated in the same direction in all diseases. Upon validation, commonalities in transcriptomic changes were restricted to comparisons between severe asthma and MDD or PTSD.

In keeping with literature indicating a close association with regards to comorbidity and reciprocal enhancement of symptom severity ${ }^{17,18}$, our crossdisease comparisons found the greatest transcriptomic level similarities between severe asthma and PTSD.

With regard to commonly differentially expressed genes we found ORMDL3 to be downregulated in the blood of both PTSD and severe asthma subjects. ORMDL 3 codes for a protein called "ORMDL sphingolipid biosynthesis regulator 3 " which resides in the endoplasmic reticulum and is a regulator of sphingolipid synthesis ${ }^{22}$. ORMDL3 requires precise expression to function correctly - under normal conditions it inhibits the rate limiting enzyme of sphingolipid biosynthesis, serine palmitoyltransferase (SPT) ${ }^{22}$. Downstream of uninhibited SPT activity, ceramide - the central sphingolipid metabolite - is produced and transported to the golgi ${ }^{23}$. Therefore, a knockdown of ORMDL3 can result in an abundance of ceramide ${ }^{24}$. When slightly overexpressed, ORMDL3 leads to a dearth of ceramide, however, when highly overexpressed, ORMDL3 increases ceramide biosynthesis through the alternate, recycling/salvage pathway ${ }^{25,23}$.

Numerous GWAS have identified ORMDL3 as a potential susceptibility gene for asthma and polymorphisms controlling ORMDL3 expression have been associated with both asthma occurrence and exacerbation $26,27,28,29,30,31,32,33$

However, the mechanistic contribution of ORMDL3 to the pathogenesis of asthma remains unclear and experimental evidence suggests the relationship between ORMDL3 and asthma is complex. Studies in animal models of allergic airway inflammation have indicated that overexpression of ORMDL3 leads to increased ceramide levels and the accompanying ER stress leads to characteristic features of asthma including increased mucus production, an exacerbated inflammatory response, and airway hyperresponsiveness. Correspondingly, downregulation of 
ORMDL3 expression, and decreased ceramide levels, were demonstrated to significantly ameliorate asthmatic symptoms in a mouse model $34,35,36,37,38,39$. Furthermore, the expression of ORMDL3 in eosinophils seems to play a role in recruitment, attachment and activation of eosinophils in asthma ${ }^{40}$. However, seemingly conflicting evidence suggests that decreased expression of ORMDL3 can also promote asthma symptoms. Selective knockdown of ORMDL3 in lung epithelial cells leads to airway hyperresponsiveness ${ }^{41}$, while downregulation of ORMDL3 in mast cells, cells key to asthma pathogenesis, enhances antigen mediated expression of proinflammatory cytokines and production of prostaglandin D2 and promotes mast cell driven inflammation in vivo ${ }^{42}$.

While, to our knowledge there have been no studies associating ORMDL3 and PTSD, ceramide is a precursor for complex sphingolipids that are highly abundant in neural cellular membranes and are regulators of brain homeostasis ${ }^{43}$. Ceramide has also been shown to promote stress-induced depression-like behaviour in mice, and intervention with drugs that reduce hippocampal ceramide (amitriptyline and fluoxetine) rescued those behaviours ${ }^{44,45}$.

Upregulated in the blood of severe asthma and PTSD subjects were mRNA encoding Syntaxin 8 (STX8), and Rho GTPase Activating Protein 24 (ARHGAP24). STX8 is a t-SNARE protein (target soluble N-ethylmaleimide-sensitive factor attachment protein receptor) involved in diverse vesicle docking and membrane fusion events. STX8 has been demonstrated to regulate the function of receptors and ion channels, including TrkA and CFTR. The TrkA receptor is transported from the golgi to the plasma membrane by STX8, a process which with nerve growth factor (NGF) stimulation promotes downstream TrkA signalling ${ }^{46}$. Interestingly, higher levels of TrkA expression have been identified in patients with allergic asthma ${ }^{47}$, and although its role in asthma has not been fully elucidated, there are several proposed mechanisms by which neurotrophin signaling exacerbates asthma ${ }^{48}$. Some evidence suggests neurotrophin signaling may modulate airway hyperactivity and bronchonconstrictor release, enhancement of airway contractility, as well as airway remodelling $48,49,50$. TrkA has also been previously implicated in PTSD, as NGF signalling via TrkA alleviated stress induced PTSD-like symptoms in mice ${ }^{51}$. In contrast to enhancing TrkA signaling, STX8 also interacts with CFTR to inhibit function and trafficking to the cell surface ${ }^{52}$. CFTR is largely studied in relation to cystic fibrosis, however, impaired function of this ion channel has been associated with more severe or difficult to treat asthma $53,54,55$. While to our knowledge there has been no suggested relationship between CFTR and PTSD, the ion channel is expressed throughout the central nervous system ${ }^{56}$.

ARHGAP24 converts the Rac-type GTPase into its inactive GDP-bound state which, downstream of Rho, suppresses actin remodelling ${ }^{57}$. Increased activation of RhoA/Rho-kinase is associated with airway hyper-responsiveness and smooth muscle contraction in asthma ${ }^{58}$. Cerebral RhoA activation is known to enhance fear memory which may have implications for PTSD ${ }^{59}$. So, in both asthma and PTSD, increased Rho activity is associated with increased pathology. It is curious then, that we find an inhibitor of its downstream activity differentially overexpressed in the blood of the diseased subjects. The reason for this would have to be elucidated by further research.

Other genes commonly downregulated in severe asthma and PTSD were Protein Tyrosine Phosphatase 4A3 (PTP4A3), known for its role in stimulating progression from $\mathrm{G} 1$ to $S$ phase in mitosis ${ }^{60}$; Shisa Family Member 4 (SHISA4), a transmembrane scaffold/adaptor protein ${ }^{61}$; and Tubulin Polymerization Promoting Protein Family Member 3 (TPPP3), a regulator of microtubule dynamics ${ }^{62}$. To our knowledge, none of these proteins have previously been associated with asthma or PTSD and their identification here may warrant further investigation.

Neither ORMDL3, STX8, nor ARHGAP24 are discussed by Bigler et al., (2017) ${ }^{63}$ in relation to the asthma datasets; nor are they identified in the PTSD dataset by Rusch et al., (2019) ${ }^{64}$. ARHGAP24 is discussed briefly in regards to PTSD in the validation data set, (Kuan et al., 2017) ${ }^{65}$ as being a member of the PTSD-associated actin cytoskeleton pathway.

Although Leday et al., (2018) ${ }^{66}$ identified 165 differentially expressed genes using their full dataset, we found no differentially expressed genes when we used only batch 1 of the dataset. PCA revealed that the dataset ${ }^{66}$ formed distinct groups along batches and to ensure that differences in gene expression could only be explained by MDD, rather than by batch differences, we chose to include only one batch of data in our analysis. While there were no shared changes in expression of individual genes, a number of gene sets were commonly upregulated in severe asthma and MDD.

One of the gene sets "GSE34205_HEALTHY_VS_RSV_INF_INFANT_PBMC_DN" refers to a list of genes found to be more highly expressed in peripheral blood mononuclear cells (PBMC) of infants with RSV (Respiratory syncytial virus) bronchiolitis ${ }^{67}$ when compared to those of healthy subjects. We also found that "GSE34205_HEALTHY_VS_FLU_INF_INFANT_PBMC_UP," a list of genes with decreased in expression infants with acute influenza compared to PBMCs of healthy subjects, was downregulated in both MDD and severe asthma ${ }^{67}$. These 2 congruent pieces of evidence suggest that the immune signature to respiratory infection in infants is similar to the immune signature of both asthma and MDD whole blood. In human airway epithelial cells loannidis et al. (2012) ${ }^{67}$ found that comparing both influenza and RSV treatment to control exhibited DE reminiscent of a type I interferon immune signature and genes downstream of IFN- $\alpha / \beta$ were expressed abundantly in infected cells. Type I interferon signaling is known to be a contributing factor in some cases of both depression and asthma $68,69,70,71$.

Two additional gene sets we found downregulated in both MDD and severe asthma: “GSE22886_NAIVE_BCELL_VS_NEUTROPHIL_UP,” and "GSE22886_NEUTROPHIL_VS_MONOCYTE_DN" were both compiled by Abbas et al. (2005) 72 to identify patterns in immune cell-specific expression in order to identify states of activation. The gene sets we identified as being underexpressed in MDD and severe asthma can be congruently 
explained by a reduction of neutrophil specific gene expression, or by an increase in naive B-cell and monocyte specific gene expression. The latter is perhaps more likely as neutrophils have been demonstrated to be activated in patients with MDD and asthma ${ }^{73,74}$. Furthermore, $\mathrm{B}$ cell homeostasis is altered in individuals with MDD and B cells play a crucial role in regulating the hyperactivity of airways in asthma ${ }^{75,76,77,78}$. Likewise, there is generally increased activity and larger numbers of monocytes in MDD and asthma compared to healthy subjects ${ }^{79,80,81,82}$. This highlights the possibility of enhanced B cell and monocyte activity playing a key role in comorbid asthma and MDD.

Despite MDD being a major comorbidity in PTSD, and 94 immune signature gene sets commonly upregulated between the exploration datasets, no genes or gene sets were validated in this study when comparing MDD and PTSD. However, the neurobiology of the link between PTSD and MDD is unclear and it is entirely feasible that similarities in gene expression between the disorders is restricted to the CNS and are undetectable in the blood.

It is notable that there were no validated genes or gene sets in common between mild/moderate asthma and either of the mental health disorders. This finding is consistent with the phenomenon that mental health disorders such as PTSD and MDD are correlated with more severe disease outcomes $^{83}$. It may be that activation of specific genes or pathways that are involved in MDD or PTSD are also factors that contribute to the development of more severe asthma. In this regard, there is evidence to suggest that antidepressant treatment improved asthma symptoms in severe but not mild asthmatics with co-morbid depression.

Overall, with six parallel DGE analyses and GSEA on whole blood gene expression, we identified genes and gene set expression that potentially links severe asthma to both PTSD and MDD. The gene sets commonly regulated between asthma and MDD, support previously suggested links between inflammation related immune factors and the two disorders ${ }^{7}$. Epidemiological evidence indicating that PTSD has a stronger association with asthma than other chronic inflammatory disease ${ }^{84,85}$ suggests that the relationship is driven by more than common immune factors. Here we identify 6 genes ( 2 upregulated in disease and 4 downregulated) being differentially expressed in both PTSD and asthma. Of particular note, our results identify mechanisms involving ceramide biosynthesis and SNARE regulated signaling pathways as potential targets for future research aimed at understanding both the relationship between PTSD and asthma and the pathophysiology of the individual disorders.

\section{Methods:}

\section{Obtaining and Preprocessing Datasets:}

Data were downloaded from the Gene Expression Omnibus (GEO) repository and preprocessed using the methods described by the respective authors associated with each dataset (Table 3). Rusch et al., (2019) ${ }^{64}$ measured blood mRNA military service members, with and without PTSD. Only samples from the first time-point collection, rather than the follow up collection, were selected for analysis. Kuan et al., (2017) ${ }^{65}$ measured blood mRNA in world trade center responders with PTSD currently, never, and in the past. Samples collected from subjects who never had PTSD or had PTSD at the time of the collection were selected for further analysis. Leday et al., (2017) ${ }^{66}$ pooled human blood mRNA data from two depression studies: the "Janssen-Brain Resource Company "study, and the "GlaxoSmithKline-High-Throughput Disease-specific target Identification Program" study into subjects with MDD, and without. Batch 1 of this dataset was used as the exploration cohort for MDD and batch 2 was excluded because these batches formed distinct groups in principal component analysis (PCA), making them inappropriate for use in determining differences between MDD and healthy subjects. Spijker et al., (2010) ${ }^{86}$ collected blood from subjects with and without MDD and stimulated expression with lipopolysaccharide (LPS) treatment prior to mRNA isolation, measurement and differential expression analysis. The Unbiased Biomarkers for the Prediction of Respiratory Disease Outcomes (U-BIOPRED) study dataset (Bigler et al., 2017) ${ }^{63}$ measured blood mRNA in subjects with moderate (lung function tests are $60-80 \%$ of expected value), severe (lung function tests are $<60 \%$ of expected value), and no asthma. We randomly divided this dataset into an exploration and a validation cohort at a 2:1 ratio. Low expressed genes were filtered out prior to TMM normalization of the RNAseq dataset as it is more sensitive due to its single nucleotide resolution ${ }^{87,88}$. This was performed using the edgeR packages 'filterByExpr' function ${ }^{89}$.

Principal component analysis (PCA) was done in base R and visualized using ggplot $2^{90}$. Venn diagrams were generated using the VennDiagram R package ${ }^{91}$.

\section{Differential Gene Expression:}

Each dataset, including the split asthma datasets for both severe and moderate asthma, underwent differential gene expression analysis individually, comparing their disease to the respective control group (the non-disease group) from the same study. Analysis was performed using the limma package (Ritchie et al., 201592) in R and genes were considered to be differentially expressed with an adjusted $p$-value $<0.05$ and $|F C| \geq 1.5$.

\section{Gene Set Enrichment Analysis:}

3 MSigDB collections of gene sets (v7.4) were downloaded from the GSEA website (https://www.gsea-msigdb.org/gsea/msigdb/index.jsp): Hallmark - well-defined biological states or processes, C2 - curated gene sets from PubMed publications and online pathway databases (including KEGG), and C7 - immunologic signature gene sets representative of immune and cell states. 
Fold change values generated by the differential expression analysis of diseased subjects vs healthy subjects were compared to each of the 3 collections via their entrez gene IDs using the Gage package in $\mathrm{R}^{93}$. Gage uses the differential expression output of all genes, not just those with significant fold change or $\mathrm{p}$-value. Barcode plots were generated using barcodeplot() function (limma package). Volcano plots were generated using the R package 'EnhancedVolcano'94.

\section{Declarations:}

\section{Acknowledgements:}

This work was supported by funding from the Natural Sciences and Engineering Research Council of Canada (NSERC) The funding agency had no role in study design; in the collection, analysis and interpretation of data; in the writing of the report; or in the decision to submit the article for publication.

P.F. is the AstraZeneca Canada Inc., Chair in Asthma and Obstructive Lung Disease.

Affiliations:

McMaster University, Hamilton, Ontario, Canada

Sandor Haas-Neill, Anna Dvorkin-Gheva

\section{Brain Body Institute, St. Joseph's Hospital, Hamilton, Ontario, Canada}

Sandor Haas-Neill

\section{Department of Medicine, Division of Pulmonary Medicine, University of Alberta, Edmonton, Alberta, Canada}

Paul Forsythe

Author Contributions:

S.H-N. \& P.F. conceived and designed the study. S.H-N Collated the data, designed and conducted the analysis, constructed the figures and wrote the first draft of the manuscript. A.DG. contributed to bioinformatics and edited the manuscript. S.H-N and P.F. wrote and edited the final manuscript, which was reviewed before submission by all authors.

\section{Additional Information:}

Competing Interests:

The authors declare no competing interests.

\section{References:}

1. Vos, T. et al. Global burden of 369 diseases and injuries in 204 countries and territories, 1990-2019: a systematic analysis for the Global Burden of Disease Study 2019. Lancet 396, 1204-1222 (2020).

2. Masoli, M., Fabian, D., Holt, S. \& Beasley, R. The global burden of asthma: executive summary of the GINA Dissemination Committee Report. Allergy 59, 469-478 (2004).

3. Janssens, T. \& Ritz, T. Perceived triggers of asthma: key to symptom perception and management. Clin. Exp. Allergy 43, 1000-1008 (2013).

4. Asher, I. \& Pearce, N. Global burden of asthma among children. Int. J. Tuberc. Lung Dis. 18, 1269-1278 (2014).

5. Wisnivesky, J. P. et al. Comorbid posttraumatic stress disorder and major depressive disorder are associated with asthma morbidity among World Trade Center workers. Ann. Allergy, Asthma Immunol. 126, 278-283 (2021).

6. Goodwin, R. D., Fischer, M. E. \& Goldberg, J. A Twin Study of Post-Traumatic Stress Disorder Symptoms and Asthma. Am. J. Respir. Crit. Care Med. 176, 983-987 (2007).

7. Jiang, M., Qin, P. \& Yang, X. Comorbidity between depression and asthma via immune-inflammatory pathways: A meta-analysis. J. Affect. Disord. 166, 22-29 (2014).

8. Katon, W. et al. The Prevalence of DSM-IV Anxiety and Depressive Disorders in Youth with Asthma Compared with Controls. J. Adolesc. Heal. 41, 455-463 (2007).

9. Caramanica, K., Brackbill, R. M., Liao, T. \& Stellman, S. D. Comorbidity of 9/11-Related PTSD and Depression in the World Trade Center Health Registry 10-11 Years Postdisaster. J. Trauma. Stress 27, 680-688 (2014).

10. Rytwinski, N. K., Scur, M. D., Feeny, N. C. \& Youngstrom, E. A. The Co-Occurrence of Major Depressive Disorder Among Individuals With Posttraumatic Stress Disorder: A Meta-Analysis. J. Trauma. Stress 26, 299-309 (2013). 
11. Kennedy, S. H. Core symptoms of major depressive disorder: relevance to diagnosis and treatment. Dialogues Clin. Neurosci. 10, 271-277 (2008).

12. Tedner, S. G., Lundholm, C., Olsson, H. \& Almqvist, C. Depression or anxiety in adult twins is associated with asthma diagnosis but not with offspring asthma. Clin. Exp. Allergy 46, 803-812 (2016).

13. Hasler, G. et al. Asthma and Panic in Young Adults. Am. J. Respir. Crit. Care Med. 171, 1224-1230 (2005).

14. To, T. et al. Mental Health Services Claims and Adult Onset Asthma in Ontario, Canada. J. Allergy Clin. Immunol. Pract. 5, 1388-1393.e3 (2017).

15. Vargas, P. A. Spreading the Word: Comorbidity of Asthma and Depression Is Not Just the Product of a Vulnerable Personality. J. Allergy Clin. Immunol. Pract. 8, 208-209 (2020).

16. Lancaster, C., Teeters, J., Gros, D. \& Back, S. Posttraumatic Stress Disorder: Overview of Evidence-Based Assessment and Treatment. J. Clin. Med. 5, 105 (2016).

17. Xu, K. Y. et al. Determinants of asthma morbidity in World Trade Center rescue and recovery workers. Ann. Allergy, Asthma Immunol. 117, 568570 (2016)

18. Chung, M. C., Rudd, H. \& Wall, N. Posttraumatic stress disorder following asthma attack (post-asthma attack PTSD) and psychiatric comorbidity: The impact of alexithymia and coping. Psychiatry Res. 197, 246-252 (2012).

19. Zhu, Z. et al. Shared genetics of asthma and mental health disorders: a large-scale genome-wide cross-trait analysis. Eur. Respir. J. 54, 1901507 (2019).

20. Cao, H., Li, S., Baranova, A. \& Zhang, F. Shared Genetic Liability Between Major Depressive Disorder and Atopic Diseases. Front. Immunol. 12, (2021).

21. Nievergelt, C. M. et al. International meta-analysis of PTSD genome-wide association studies identifies sex- and ancestry-specific genetic risk loci. Nat. Commun. 10, 4558 (2019).

22. Breslow, D. K. et al. Orm family proteins mediate sphingolipid homeostasis. Nature 463, 1048-1053 (2010).

23. James, B., Milstien, S. \& Spiegel, S. ORMDL3 and allergic asthma: From physiology to pathology. J. Allergy Clin. Immunol. 144, 634-640 (2019).

24. Cai, L. et al. ORMDL proteins regulate ceramide levels during sterile inflammation. J. Lipid Res. 57, 1412-1422 (2016).

25. Oyeniran, C. et al. Aberrant ORM (yeast)-like protein isoform 3 (ORMDL3) expression dysregulates ceramide homeostasis in cells and ceramide exacerbates allergic asthma in mice. J. Allergy Clin. Immunol. 136, 1035-1046.e6 (2015).

26. Ferreira, M. A. R. et al. Association between ORMDL3, IL1RL1 and a deletion on chromosome 17q21 with asthma risk in Australia. Eur. J. Hum. Genet. 19, 458-464 (2011).

27. Moffatt, M. F. et al. Genetic variants regulating ORMDL3 expression contribute to the risk of childhood asthma. Nature 448, 470-473 (2007).

28. Choudhry, S. et al. Genome-wide screen for asthma in Puerto Ricans: evidence for association with 5q23 region. Hum. Genet. 123, 455-468 (2008).

29. Himes, B. E. et al. Genome-wide Association Analysis Identifies PDE4D as an Asthma-Susceptibility Gene. Am. J. Hum. Genet. 84, 581-593 (2009).

30. Hancock, D. B. et al. Genome-Wide Association Study Implicates Chromosome 9q21.31 as a Susceptibility Locus for Asthma in Mexican Children. PLoS Genet. 5, e1000623 (2009).

31. Sleiman, P. M. A. et al. Variants of DENND1B Associated with Asthma in Children. N. Engl. J. Med. 362, 36-44 (2010).

32. Mathias, R. A. et al. A genome-wide association study on African-ancestry populations for asthma. J. Allergy Clin. Immunol. 125, 336-346.e4 (2010).

33. Li, X. et al. Genome-wide association study of asthma identifies RAD50-IL13 and HLA-DR/DQ regions. J. Allergy Clin. Immunol. 125, 328-335.e11 (2010).

34. Oyeniran, C. et al. Aberrant ORM (yeast)-like protein isoform 3 (ORMDL3) expression dysregulates ceramide homeostasis in cells and ceramide exacerbates allergic asthma in mice. J. Allergy Clin. Immunol. 136, 1035-1046.e6 (2015).

35. Masini, E. et al. Ceramide: a Key Signaling Molecule in a Guinea Pig Model of Allergic Asthmatic Response and Airway Inflammation. J. Pharmacol. Exp. Ther. 324, 548-557 (2008).

36. Petrache, I. et al. Ceramide Synthases Expression and Role of Ceramide Synthase- 2 in the Lung: Insight from Human Lung Cells and Mouse Models. PLoS One 8, e62968 (2013).

37. Spassieva, S. D., Mullen, T. D., Townsend, D. M. \& Obeid, L. M. Disruption of ceramide synthesis by CerS2 down-regulation leads to autophagy and the unfolded protein response. Biochem. J. 424, 273-283 (2009).

38. Epstein, S. et al. Activation of the unfolded protein response pathway causes ceramide accumulation in yeast and INS-1E insulinoma cells. J. Lipid Res. 53, 412-420 (2012).

39. Choi, S. et al. Myristate-induced endoplasmic reticulum stress requires ceramide synthases $5 / 6$ and generation of C14-ceramide in intestinal epithelial cells. FASEB J. 32, 5724-5736 (2018).

40. Ha, S. G. et al. ORMDL3 promotes eosinophil trafficking and activation via regulation of integrins and CD48. Nat. Commun. 4, 2479 (2013). 
41. Miller, M. et al. Cutting Edge: Targeting Epithelial ORMDL3 Increases, Rather than Reduces, Airway Responsiveness and Is Associated with Increased Sphingosine-1-Phosphate. J. Immunol. 198, 3017-3022 (2017).

42. Bugajev, V. et al. Negative regulatory roles of ORMDL3 in the FcERI-triggered expression of proinflammatory mediators and chemotactic response in murine mast cells. Cell. Mol. Life Sci. 73, 1265-1285 (2016).

43. Mencarelli, C. \& Martinez-Martinez, P. Ceramide function in the brain: when a slight tilt is enough. Cell. Mol. Life Sci. 70, 181-203 (2013).

44. Kornhuber, J. \& Gulbins, E. New Molecular Targets for Antidepressant Drugs. Pharmaceuticals 14, 894 (2021).

45. Gulbins, E. et al. Acid sphingomyelinase-ceramide system mediates effects of antidepressant drugs. Nat. Med. 19, 934-938 (2013).

46. Chen, B. et al. Syntaxin 8 Modulates the Post-synthetic Trafficking of the TrkA Receptor and Inflammatory Pain Transmission*. J. Biol. Chem. 289, 19556-19569 (2014).

47. Piedimonte, G. Contribution of neuroimmune mechanisms to airway inflammation and remodeling during and after respiratory syncytial virus infection. Pediatr. Infect. Dis. J. 22, S66-S75 (2003).

48. Prakash, Y. et al. Neurotrophins in lung health and disease. Expert Rev. Respir. Med. 4, 395-411 (2010).

49. Prakash, Y. S., Thompson, M. A. \& Pabelick, C. M. Brain-Derived Neurotrophic Factor in TNF-a Modulation of Ca 2+ in Human Airway Smooth Muscle. Am. J. Respir. Cell Mol. Biol. 41, 603-611 (2009).

50. Micera, A. et al. Nerve growth factor displays stimulatory effects on human skin and lung fibroblasts, demonstrating a direct role for this factor in tissue repair. Proc. Natl. Acad. Sci. 98, 6162-6167 (2001).

51. Feng, D.-Y. et al. Nerve growth factor against PTSD symptoms: Preventing the impaired hippocampal cytoarchitectures. Prog. Neurobiol. 184, 101721 (2020).

52. Bilan, F. et al. Syntaxin 8 impairs trafficking of cystic fibrosis transmembrane conductance regulator (CFTR) and inhibits its channel activity. J. Cell Sci. 117, 1923-1935 (2004).

53. Dahl, M., Tybærg-Hansen, A., Lange, P. \& Nordestgaard, B. G. $\Delta$ F508 heterozygosity in cystic fibrosis and susceptibility to asthma. Lancet 351, 1911-1913 (1998).

54. Dahl, M., Nordestgaard, B. G., Lange, P. \& Tybjaerg-Hansen, A. Fifteen-year follow-up of pulmonary function in individuals heterozygous for the cystic fibrosis phenylalanine-508 deletion. J. Allergy Clin. Immunol. 107, 818-23 (2001).

55. Douros, K. et al. Asthma and pulmonary function abnormalities in heterozygotes for cystic fibrosis transmembrane regulator gene mutations. Int. J. Clin. Exp. Med. 1, 345-9 (2008).

56. Reznikov, L. R. Cystic Fibrosis and the Nervous System. Chest 151, 1147-1155 (2017).

57. Lavelin, I. \& Geiger, B. Characterization of a Novel GTPase-activating Protein Associated with Focal Adhesions and the Actin Cytoskeleton. J. Biol. Chem. 280, 7178-7185 (2005).

58. Chiba, Y. et al. Involvement of RhoA-mediated Ca2+ sensitization in antigen-induced bronchial smooth muscle hyperresponsiveness in mice. Respir. Res. 6, 4 (2005).

59. Diana, G. et al. Enhancement of learning and memory after activation of cerebral Rho GTPases. Proc. Natl. Acad. Sci. 104, 636-641 (2007).

60. den Hollander, P. et al. Phosphatase PTP4A3 Promotes Triple-Negative Breast Cancer Growth and Predicts Poor Patient Survival. Cancer Res. 76, 1942-1953 (2016).

61. Pei, J. \& Grishin, N. V. Unexpected diversity in Shisa-like proteins suggests the importance of their roles as transmembrane adaptors. Cell. Signal. 24, 758-769 (2012).

62. Oláh, J. et al. Microtubule-Associated Proteins with Regulatory Functions by Day and Pathological Potency at Night. Cells 9,357 (2020).

63. Bigler, J. et al. A Severe Asthma Disease Signature from Gene Expression Profiling of Peripheral Blood from U-BIOPRED Cohorts. Am. J. Respir. Crit. Care Med. 195, 1311-1320 (2017).

64. Rusch, H. L. et al. Gene expression differences in PTSD are uniquely related to the intrusion symptom cluster: A transcriptome-wide analysis in military service members. Brain. Behav. Immun. 80, 904-908 (2019).

65. Kuan, P.-F. et al. Gene expression associated with PTSD in World Trade Center responders: An RNA sequencing study. Transl. Psychiatry 7, 1297 (2017).

66. Leday, G. G. R. et al. Replicable and Coupled Changes in Innate and Adaptive Immune Gene Expression in Two Case-Control Studies of Blood Microarrays in Major Depressive Disorder. Biol. Psychiatry 83, 70-80 (2018).

67. Ioannidis, I. et al. Plasticity and Virus Specificity of the Airway Epithelial Cell Immune Response during Respiratory Virus Infection. J. Virol. 86, 5422-5436 (2012).

68. Franscina Pinto, E. \& Andrade, C. Interferon-Related Depression: A Primer on Mechanisms, Treatment, and Prevention of a Common Clinical Problem. Curr. Neuropharmacol. 14, 743-748 (2016).

69. Zheng, L.-S. et al. Mechanisms for Interferon-a-Induced Depression and Neural Stem Cell Dysfunction. Stem Cell Reports 3, 73-84 (2014).

70. Rich, H. E., Antos, D., Melton, N. R., Alcorn, J. F. \& Manni, M. L. Insights Into Type I and III Interferons in Asthma and Exacerbations. Front. Immunol. 11, (2020). 
71. Gomez, J. L., Diaz, M. P., Nino, G. \& Britto, C. J. Impaired type I interferon regulation in the blood transcriptome of recurrent asthma exacerbations. BMC Med. Genomics 11, 21 (2018).

72. Abbas, A. R. et al. Immune response in silico (IRIS): immune-specific genes identified from a compendium of microarray expression data. Genes Immun. 6, 319-331 (2005).

73. et al. Neutrophil-lymphocyte ratio in patients with major depressive disorder undergoing no pharmacological therapy. Neuropsychiatr. Dis. Treat. 2253 (2015) doi:10.2147/NDT.S89470.

74. Gao, H., Ying, S. \& Dai, Y. Pathological Roles of Neutrophil-Mediated Inflammation in Asthma and Its Potential for Therapy as a Target. J. Immunol. Res. 2017, 1-12 (2017).

75. Ahmetspahic, D. et al. Altered B Cell Homeostasis in Patients with Major Depressive Disorder and Normalization of CD5 Surface Expression on Regulatory B Cells in Treatment Responders. J. Neuroimmune Pharmacol. 13, 90-99 (2018).

76. Habener, A. et al. B cells are crucial in the regulation of airway hyperreactivity in an experimental model of asthma. in Allergy and immunology PA4362 (European Respiratory Society, 2019). doi:10.1183/13993003.congress-2019.PA4362.

77. Ghosh, S. et al. B lymphocytes regulate airway granulocytic inflammation and cytokine production in a murine model of fungal allergic asthma. Cell. Mol. Immunol. 12, 202-212 (2015).

78. De Vooght, V. et al. B-lymphocytes as Key Players in Chemical-Induced Asthma. PLoS One 8, e83228 (2013).

79. Alvarez-Mon, M. A. et al. Abnormal Distribution and Function of Circulating Monocytes and Enhanced Bacterial Translocation in Major Depressive Disorder. Front. Psychiatry 10, (2019).

80. Seidel, A. et al. Major depressive disorder is associated with elevated monocyte counts. Acta Psychiatr. Scand. 94, 198-204 (1996).

81. Niessen, N. M. et al. Neutrophilic asthma features increased airway classical monocytes. Clin. Exp. Allergy 51, 305-317 (2021).

82. Tanizaki, Y. et al. Numerical changes in blood monocytes in bronchial asthma. Acta Med. Okayama 36, 341-8 (1982).

83. Kewalramani, A., Bollinger, M. E. \& Postolache, T. T. Asthma and Mood Disorders. Int. J. Child Health Hum. Dev. 1, 115-123 (2008).

84. O'Toole, B. I. \& Catts, S. V. Trauma, PTSD, and physical health: An epidemiological study of Australian Vietnam veterans. J. Psychosom. Res. 64, 33-40 (2008).

85. Allgire, E., McAlees, J. W., Lewkowich, I. P. \& Sah, R. Asthma and posttraumatic stress disorder (PTSD): Emerging links, potential models and mechanisms. Brain. Behav. Immun. 97, 275-285 (2021).

86. Spijker, S. et al. Stimulated Gene Expression Profiles as a Blood Marker of Major Depressive Disorder. Biol. Psychiatry 68, 179-186 (2010).

87. Wilhelm, B. T. et al. Dynamic repertoire of a eukaryotic transcriptome surveyed at single-nucleotide resolution. Nature 453, 1239-1243 (2008).

88. Xu, X. et al. Parallel comparison of Illumina RNA-Seq and Affymetrix microarray platforms on transcriptomic profiles generated from 5-azadeoxy-cytidine treated HT-29 colon cancer cells and simulated datasets. BMC Bioinformatics 14, S1 (2013).

89. Robinson, M. D., McCarthy, D. J. \& Smyth, G. K. edgeR: a Bioconductor package for differential expression analysis of digital gene expression data. Bioinformatics 26, 139-140 (2010).

90. H, W. ggplot2: Elegant Graphics for Data Analysis. (Springer-Verlag, 2016).

91. Chen, H. \& Boutros, P. C. VennDiagram: a package for the generation of highly-customizable Venn and Euler diagrams in R. BMC Bioinformatics 12, 35 (2011).

92. Ritchie, M. E. et al. limma powers differential expression analyses for RNA-sequencing and microarray studies. Nucleic Acids Res. 43, e47-e47 (2015).

93. Luo, W., Friedman, M. S., Shedden, K., Hankenson, K. D. \& Woolf, P. J. GAGE: generally applicable gene set enrichment for pathway analysis. BMC Bioinformatics 10, 161 (2009).

94. Blighe, K; Rana, S; Lewis, M. EnhancedVolcano: Publication-ready volcano plots with enhanced colouring and labeling. (2018).

\section{Figures}




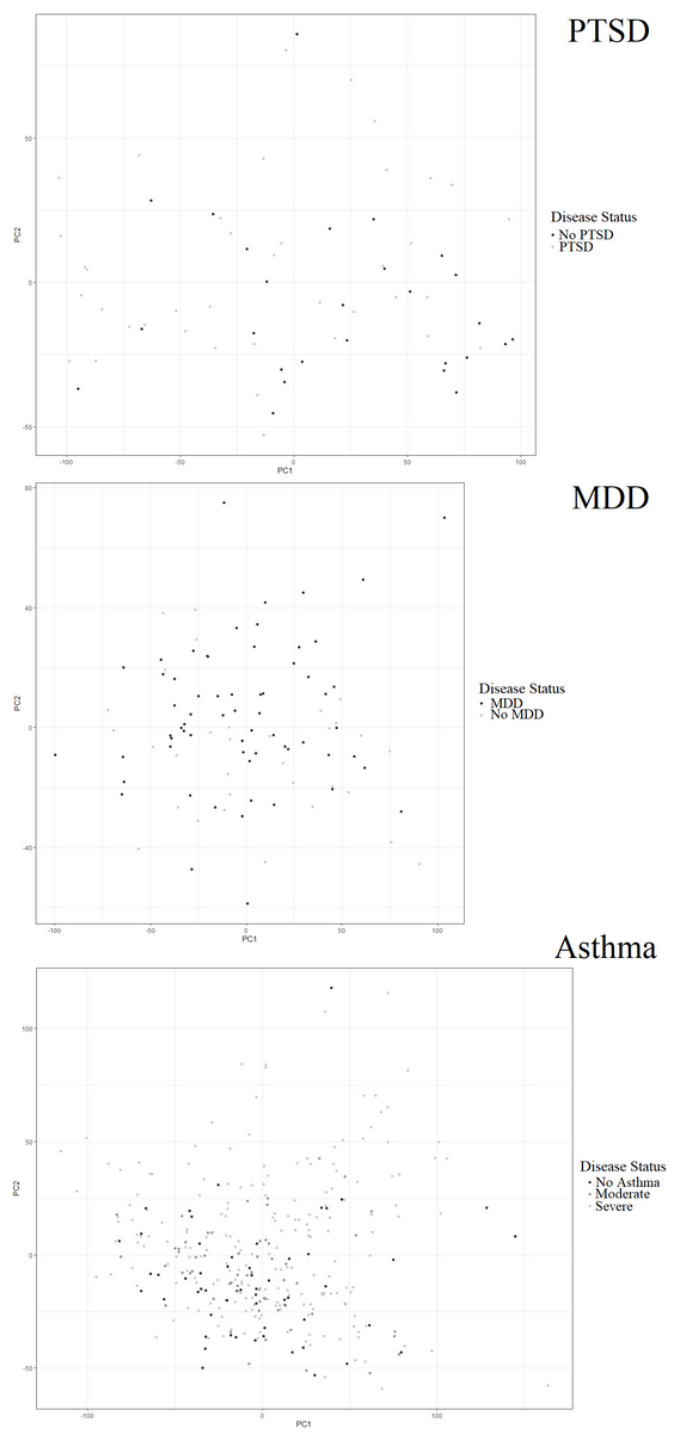

\section{Figure 1}

Principal component analysis (PCA) showing PC1 and PC2 in each of the 3 disease exploration datasets. 


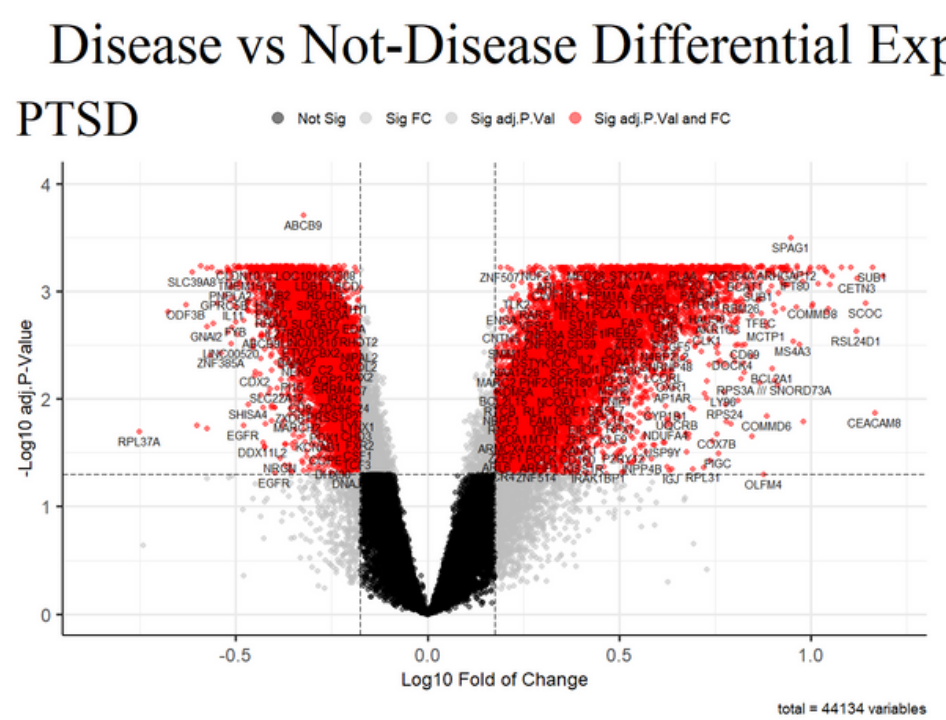

pression (Adj P-Value $\leq 0.05,|F C| \geq 1.5$ )

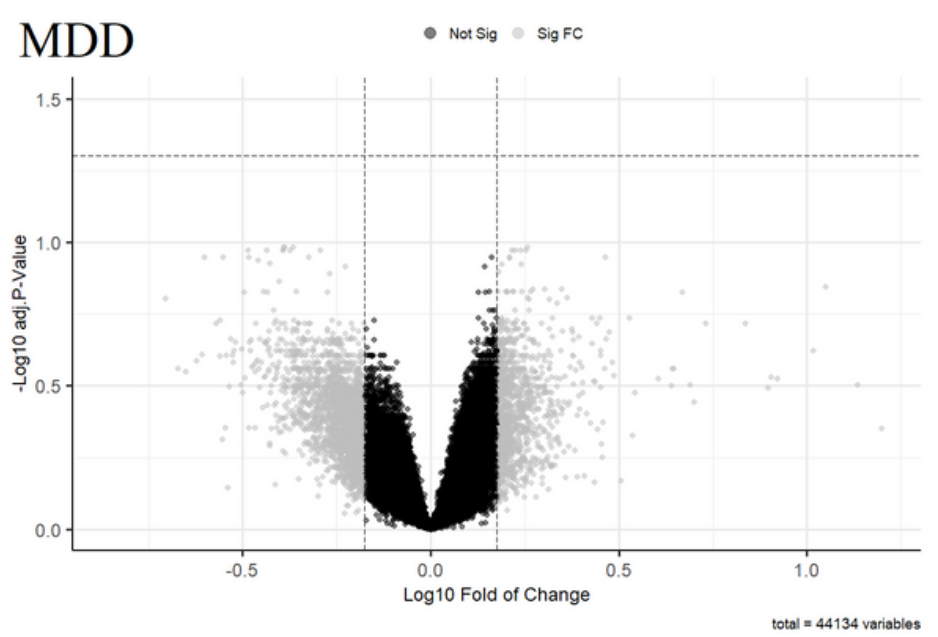

Severe Asthma

Moderate Asthma


Figure 2

mRNA from the blood of subjects with a disease (PTSD, MDD, severe asthma, and moderate asthma) were compared to blood mRNA from nondiseased subjects for each exploration cohort dataset. The vertical threshold denotes genes or transcripts that are statistically significant (adjusted pvalue $<0.05$ ) while the horizontal threshold denotes genes or transcripts with an absolute fold change greater than 1.5 . Genes or transcripts that meet none of these criteria are black, one of these criteria are grey, and both are red. The red genes, found to be significant, are also shown next to their symbols. 


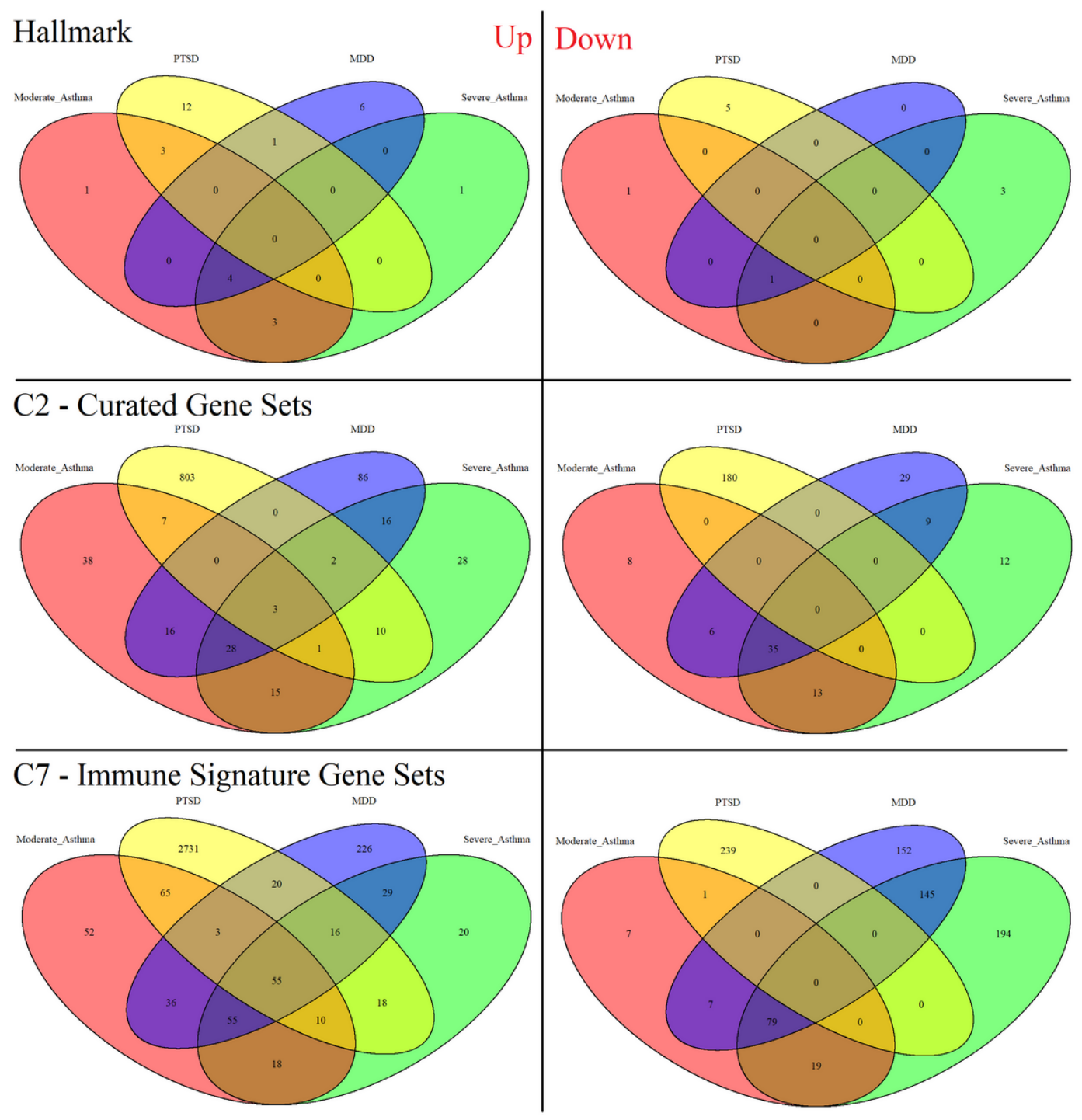

\section{Figure 3}

Gene set enrichment analysis (GSEA) showing significantly (adjusted P-value $<0.05$ ) modified pathways between all 4 exploration datasets. Labels identify the gene sets being compared to and in which direction (left = upregulated, right = downregulated).

\section{Supplementary Files}

This is a list of supplementary files associated with this preprint. Click to download.

- OnlySupplementary.docx 